\title{
Comparative Analysis of Color Reproduction of Different Order Polynomial Regression Algorithm
}

\author{
Qi Jia ${ }^{1}$, Yimin $\mathrm{Fu}^{1, *}$, Xianhui Rong ${ }^{1,2}$, Weidong $\mathrm{Xu}^{1}$ and Xuliang $\mathrm{Lv}^{1}$ \\ ${ }^{1}$ College of Field Engineering, PLA University of Science and Technology, Nanjing, China \\ ${ }^{2}$ Business School of Hohai University, Nanjing, China \\ ${ }^{*}$ Corresponding author
}

\begin{abstract}
In the visible light band, color is one of the most basic and the most important camouflage exposure symptoms. The use of color camouflage is the most basic means of resisting high-tech detection and monitoring means and precision guided weapon capture. The existing camouflage color collection mainly uses the field spectrometer and the sub-meter, but they are not easy to carry and the collection efficiency is low. This paper proposes the use of digital cameras for image acquisition, but the digital camera produces a color space of the signal is not the standard color of the color space, so the need for color reproduction, so that we can get closer to the original scene of the true color. In this paper, the color reduction is carried out by polynomial linear regression method, and the reduction effect is analyzed and evaluated. The results show that the color of the image acquired by the digital camera can accurately obtain the color of the target and the background, and provide a new method to improve the color collection efficiency in the field environment.
\end{abstract}

Keywords-color reproduction; digital camera; polynomial regression algorithm; color difference

\section{INTRODUCTION}

With the development of computer applications and image technology, photographic investigation of this means of detection play a greater role. Digital images have many advantages, can be flexibly modified, the stability of information dissemination, easy to store and repair, easy to copy the image, but also compatible with digital networks and computers [1].

In the image processing, the digital camera is a very useful tool for digital image acquisition. It can get a variety of data quickly and easily, especially in the texture and threedimensional features of the original image, the more outstanding performance. Although it has a good performance in the color image digitization process, the relevant color management of digital cameras is still a very important thing. Because the color space of the signal generated by the digital camera is a device-related color space, and is not a standard colorimetric color space. Because the value of the final digital camera is not a value based on the conversion of the deviceindependent tristimulus value from the positive color matching function, which is because the spectral sensitivity of the digital camera's color sensor is very different. Of course, the digital camera at the factory also carried out some color management processing, but this color management is based on the appearance of the image as the main basis, that is, the purpose of image acquisition is "beautiful" rather than "faithful", so the need for color restore [2] and other series of color management approach, so that we can get closer to the original scene of the true color for the camouflage target and background extraction, analysis, background census, camouflage pattern design and camouflage effect evaluation to provide data support.

\section{METHODS \& RESEARCH STEPS}

Mainly based on the color space structure of the color management method, that is directly to the camera to obtain the data correction, so that it is based on a closer to the true value of the color signal [3].

For the digital camera to obtain the data and the real data between the original calculation. And evaluate the importance of color space and multi - order polynomials in the calculation of color reproduction, and find out the optimal results. The results of the study can be applied directly to the color correction of digital camera images under the same environmental limits.

The research plan is based on the data acquisition of the digital camera and the data acquisition of the spectrometer. The data obtained by the spectrometer is taken as the standard value. The data obtained by the digital camera is taken as the measured value. The reduction model is established by polynomial, the polynomial reduction coefficient is solved, and then the regression value of the measured value is corrected to see whether the polynomial regression is effective for color reduction and the order polynomial evaluates the accuracy of its color reproduction.

The polynomial regression algorithm [4] establishes a set of equations between the color space and the target color space, and linearly combines the input primitive color space variables to fit the data of the target color space. Firstly, the coefficient matrix of the polynomial is obtained by the selected polynomial model, and then the coefficient matrix is solved to establish the reduction relation between the target color space and the original color space.

The polynomial of the polynomial regression algorithm is as follows:

First order:

$$
f(x, y, z)=a_{0}+a_{1} x+a_{2} y+a_{3} z
$$


Second order:

$f(x, y, z)=\mathrm{a}_{0}+\mathrm{a}_{1} x+\mathrm{a}_{2} y+\mathrm{a}_{3} z+\mathrm{a}_{4} x^{2}+\mathrm{a}_{5} y^{2}+\mathrm{a}_{6} z^{2}+\mathrm{a}_{7} x y+\mathrm{a}_{8} x z+\mathrm{a}_{9} y z$

Third order:

$f(x, y, z)=\mathrm{a}_{0}+\mathrm{a}_{1} x+\mathrm{a}_{2} y+\mathrm{a}_{3} z+\mathrm{a}_{4} x^{2}+\mathrm{a}_{5} y^{2}+\mathrm{a}_{6} \mathrm{z}^{2}+\mathrm{a}_{7} x y+\mathrm{a}_{8} x z+\mathrm{a}_{9} y z+\mathrm{a}_{10} x^{3}+$ $\mathrm{a}_{11} y^{3}+\mathrm{a}_{12} z^{3}+\mathrm{a}_{13} x^{2} y+\mathrm{a}_{14} x^{2} z+\mathrm{a}_{15} x y^{2}+\mathrm{a}_{16} x z^{2}+\mathrm{a}_{17} x y z+\mathrm{a}_{18} y^{2} z+\mathrm{a}_{19} y z^{2}$

Fourth order:

$f(x, y, z)=\mathrm{a}_{0}+\mathrm{a}_{1} x+\mathrm{a}_{2} y+\mathrm{a}_{3} z+\mathrm{a}_{4} x^{2}+\mathrm{a}_{5} y^{2}+\mathrm{a}_{6} z^{2}+\mathrm{a}_{7} x y+\mathrm{a}_{8} x z+\mathrm{a}_{9} y z+\mathrm{a}_{10} x^{3}+$ $\mathrm{a}_{11} y^{3}+\mathrm{a}_{12} z^{3}+\mathrm{a}_{13} x^{2} y+\mathrm{a}_{14} x^{2} z+\mathrm{a}_{15} x y^{2}+\mathrm{a}_{16} x z^{2}+\mathrm{a}_{17} x y z+\mathrm{a}_{18} y^{2} z+\mathrm{a}_{19} y z^{2}+\mathrm{a}_{2}$ ${ }_{0} x^{4}+\mathrm{a}_{21} x^{3} y+\mathrm{a}_{22} x^{3} z+\mathrm{a}_{24} x^{2} y^{2}+\mathrm{a}_{25} x^{2} y z+\mathrm{a}_{26} x y^{2} z+\mathrm{a}_{27} x y z^{2}+\mathrm{a}_{28} x y^{3}+\mathrm{a}_{29} x z^{3}$ $+a_{30} y^{4}+a_{31} y^{3} z+a_{32} y^{2} z^{2}+a_{33} y z^{3}+a_{34} z^{4}$

The condition of the polynomial regression algorithm is that the number of selected polynomials must be less than the number of samples in the color space to solve the coefficients in the polynomial. Then, into the corresponding original color space data, you can according to the system to achieve color reproduction. In the polynomial regression method, with the increase of the order, the data regression precision of the polynomial increases, and the whole image quality decreases, which is mainly caused by the decrease of the performance of the model. Therefore, the actual application of the second-order, third-order and fourth-order polynomial regression.

\section{EXPERIMENTAL DESIGN \& EXPLORATION}

The light source plays a decisive role in the formation of color. In daily life, say "see" the object, but see the object itself is not seen by the object from the reflection or transmission of the "light" .Since the color starts at the light, the color seen is affected by the characteristics of the light source used for illumination. As the experimental measurement needs to establish a reduction model with the standard value of the color measured by the spectrometer, the control variable method is used to simulate the light source of the spectrometer as much as possible under simulated field conditions. In accordance with this requirement, the spectrometer measurement using D65 light source, digital camera collection to take sunny noon at 13 o'clock under the sun light source measurement, as shown in Figure 1 below.

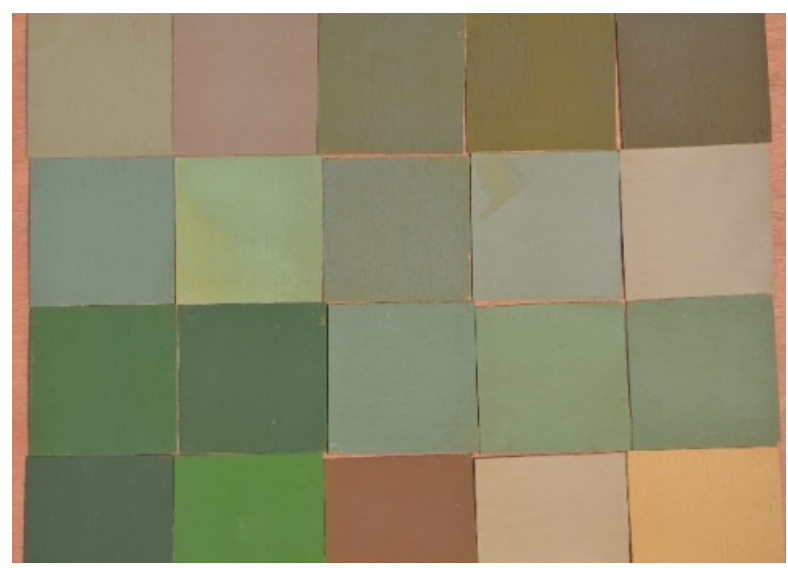

FIGURE I. DIGITAL CAMERA COLLECTION

The color plates were measured with a digital camera and a spectrometer respectively. The spectral reflectance $\mathrm{R}$ of 20 color samples was measured by a spectrometer. The S, R and CIE standard chromaticity observers' spectral tristimulus values $\mathrm{X}, \mathrm{Y}$, and $\mathrm{Z}$ functions were calculated according to the formula, and 20 color patches Light source under the brightness of the $\mathrm{XYZ}$ value, and according to literature and related information [5], we can calculate the corresponding RGB, Lab values.

Spectrometer measures the spectral reflectance of 20 color samples and the calculated RGB, XYZ, Lab values as shown in Table 1.

With the digital camera to measure the value of each color block, each time also need to measure three times, first each color sample contains the pixel data values as described above, and then the average of the three measurements again, and finally get each the color of the camera under the measured value.

The output of the digital camera image data is based on the sRGB color space, and the XYZ value and the $\mathrm{LAB}$ value of each color sample under the light source are calculated based on the relevant formula. Digital camera measured 20 color samples of RGB, LAB, XYZ value shown in Table 2.

In the Lab color space, the standard values obtained from the 20 color spectrometer measurements and the measured values collected by the digital camera were used to establish the linear equations of the fourth order polynomials respectively. The coefficients were solved respectively. The polynomial linear regression of the measured values of the digital camera was carried out. The same color of the standard value of the color calculation, plotted in the following Figure 2. 
TABLE I. SPECTRUM ANALYZER MEASURED 20 COLOR SAMPLES OF RGB, XYZ, LAB VALUE

\begin{tabular}{|c|c|c|c|c|c|}
\hline Num. & 1 & 2 & 3 & 4 & 5 \\
\hline $\mathbf{R}$ & 7.075 & 8.645 & 15.783 & 35.559 & 47.064 \\
\hline G & 8.639 & 13.793 & 9.484 & 26.728 & 29.349 \\
\hline B & 5.811 & 6.035 & 5.985 & 18.422 & 14.786 \\
\hline$X$ & 7.056 & 9.586 & 10.981 & 27.548 & 32.574 \\
\hline $\mathbf{Y}$ & 8.102 & 12.138 & 10.571 & 28.006 & 32.065 \\
\hline $\mathbf{Z}$ & 6.688 & 7.546 & 7.123 & 21.378 & 18.458 \\
\hline L & 34.195 & 41.435 & 38.848 & 59.895 & 63.397 \\
\hline a & -6.218 & 14.818 & 7.11 & 3.765 & 7.682 \\
\hline b & 7.63 & 16.875 & 13.978 & 14.608 & 26.2 \\
\hline Num. & 6 & 7 & 8 & 9 & 10 \\
\hline $\mathbf{R}$ & 7.539 & 7.09 & 15.395 & 16.973 & 15.959 \\
\hline G & 10.585 & 9.4 & 17.43 & 18.737 & 17.219 \\
\hline B & 6.087 & 6.001 & 12.492 & 12.01 & 11.43 \\
\hline $\mathrm{X}$ & 7.992 & 7.368 & 14.836 & 15.867 & 14.801 \\
\hline $\mathbf{Y}$ & 9.612 & 8.664 & 16.641 & 17.876 & 16.533 \\
\hline $\mathbf{Z}$ & 7.191 & 6.96 & 14.245 & 13.974 & 13.222 \\
\hline L & 37.137 & 35.329 & 47.804 & 49.346 & 47.666 \\
\hline a & 9.983 & 8.042 & 5.8 & 6.347 & 5.415 \\
\hline b & 10.771 & 8.529 & 8.475 & 11.783 & 10.73 \\
\hline Num. & 11 & 12 & 13 & 14 & 15 \\
\hline $\mathbf{R}$ & 14.681 & 20.81 & 14.747 & 21.55 & 33.117 \\
\hline G & 16.989 & 23.038 & 14.643 & 21.05 & 26.125 \\
\hline B & 12.856 & 14.739 & 10.328 & 16.07 & 19.534 \\
\hline $\mathbf{X}$ & 14.449 & 19.48 & 13.182 & 19.315 & 26.525 \\
\hline $\mathbf{Y}$ & 16.199 & 21.965 & 14.354 & 20.797 & 27.136 \\
\hline $\mathbf{Z}$ & 14.524 & 17.154 & 11.845 & 18.196 & 22.315 \\
\hline L & 47.235 & 53.99 & 44.737 & 52.727 & 59.1 \\
\hline a & 5.707 & 6.88 & 2.978 & 2.271 & 3.044 \\
\hline b & 6.835 & 12.653 & 9.243 & 8.331 & 11.565 \\
\hline Num. & 16 & 17 & 18 & 19 & 20 \\
\hline $\mathbf{R}$ & 23.225 & 21.824 & 13.317 & 13.036 & 11.944 \\
\hline G & 19.462 & 16.025 & 11.788 & 10.407 & 9.23 \\
\hline B & 13.639 & 12.353 & 7.679 & 5.99 & 6.029 \\
\hline $\mathbf{X}$ & 18.999 & 16.961 & 11.093 & 10.179 & 9.315 \\
\hline $\mathbf{Y}$ & 19.842 & 16.994 & 11.816 & 10.647 & 9.576 \\
\hline $\mathbf{Z}$ & 15.729 & 14.07 & 8.959 & 7.184 & 7.06 \\
\hline $\mathbf{L}$ & 51.658 & 48.252 & 40.923 & 38.98 & 37.071 \\
\hline a & 0.726 & 4.552 & 1.005 & 0.462 & 1.771 \\
\hline b & 11.71 & 9.664 & 11.152 & 13.975 & 11.153 \\
\hline
\end{tabular}

TABLE II. RGB, LAB, XYZ VALUES OF 20 COLOR SAMPLES MEASURED BY A DIGITAL CAMERA

\begin{tabular}{|c|c|c|c|c|c|}
\hline Num. & 1 & 2 & 3 & 4 & 5 \\
\hline $\mathbf{R}$ & 89 & 92 & 149 & 193 & 213 \\
\hline G & 104 & 133 & 105 & 167 & 166 \\
\hline B & 85 & 63 & 78 & 135 & 112 \\
\hline $\mathbf{X}$ & 10.799 & 13.795 & 19.092 & 40.664 & 44.6 \\
\hline $\mathbf{Y}$ & 12.68 & 19.409 & 17.043 & 40.724 & 42.588 \\
\hline $\mathbf{Z}$ & 10.477 & 7.727 & 9.505 & 28.664 & 21.231 \\
\hline $\mathbf{L}$ & 42.277 & 51.163 & 48.313 & 69.982 & 71.275 \\
\hline $\mathbf{a}$ & -9.019 & 26.728 & 15.612 & 6.15 & 12.363 \\
\hline b & 8.829 & 32.994 & 22.163 & 20.063 & 34.498 \\
\hline Num. & 6 & 7 & 8 & 9 & 10 \\
\hline $\mathbf{R}$ & 90 & 90 & 133 & 137 & 133 \\
\hline G & 116 & 110 & 145 & 148 & 142 \\
\hline B & 77 & 83 & 121 & 114 & 111 \\
\hline $\mathrm{X}$ & 11.893 & 11.446 & 23.46 & 24.168 & 22.426 \\
\hline $\mathbf{Y}$ & 15.2 & 13.95 & 26.617 & 27.713 & 25.48 \\
\hline $\mathbf{Z}$ & 9.333 & 10.278 & 22.001 & 20.006 & 18.786 \\
\hline $\mathbf{L}$ & 45.907 & 44.161 & 58.618 & 59.629 & 57.54 \\
\hline $\mathbf{a}$ & 16.75 & 12.402 & -7.984 & -9.212 & -8.014 \\
\hline b & 18.553 & 12.662 & 11.29 & 16.691 & 15.451 \\
\hline Num. & 11 & 12 & 13 & 14 & 15 \\
\hline $\mathbf{R}$ & 130 & 152 & 135 & 155 & 185 \\
\hline G & 144 & 166 & 137 & 157 & 164 \\
\hline B & 127 & 128 & 115 & 135 & 143 \\
\hline $\mathbf{X}$ & 23.21 & 30.764 & 22.249 & 30.242 & 38.677 \\
\hline $\mathbf{Y}$ & 26.224 & 35.506 & 24.28 & 32.831 & 38.848 \\
\hline $\mathbf{Z}$ & 23.927 & 25.668 & 19.745 & 27.68 & 31.469 \\
\hline $\mathbf{L}$ & 58.249 & 66.141 & 56.367 & 64.024 & 68.641 \\
\hline a & -7.51 & 10.752 & -3.768 & -3.579 & 5.692 \\
\hline b & 7.324 & 18.07 & 11.565 & 11.274 & 13.699 \\
\hline Num. & 16 & 17 & 18 & 19 & 20 \\
\hline $\mathbf{R}$ & 159 & 164 & 128 & 132 & 124 \\
\hline G & 148 & 138 & 123 & 115 & 109 \\
\hline B & 118 & 122 & 95 & 71 & 86 \\
\hline $\mathbf{X}$ & 28.47 & 28.245 & 18.244 & 16.991 & 15.641 \\
\hline $\mathbf{Y}$ & 29.858 & 27.474 & 19.581 & 17.621 & 15.894 \\
\hline $\mathbf{Z}$ & 21.418 & 22.244 & 13.654 & 8.478 & 11.057 \\
\hline $\mathbf{L}$ & 61.532 & 59.411 & 51.36 & 49.034 & 46.835 \\
\hline a & 0.359 & 8.619 & -1.914 & 1.351 & 3.164 \\
\hline b & 17.358 & 12.225 & 16.029 & 26.724 & 15.027 \\
\hline
\end{tabular}




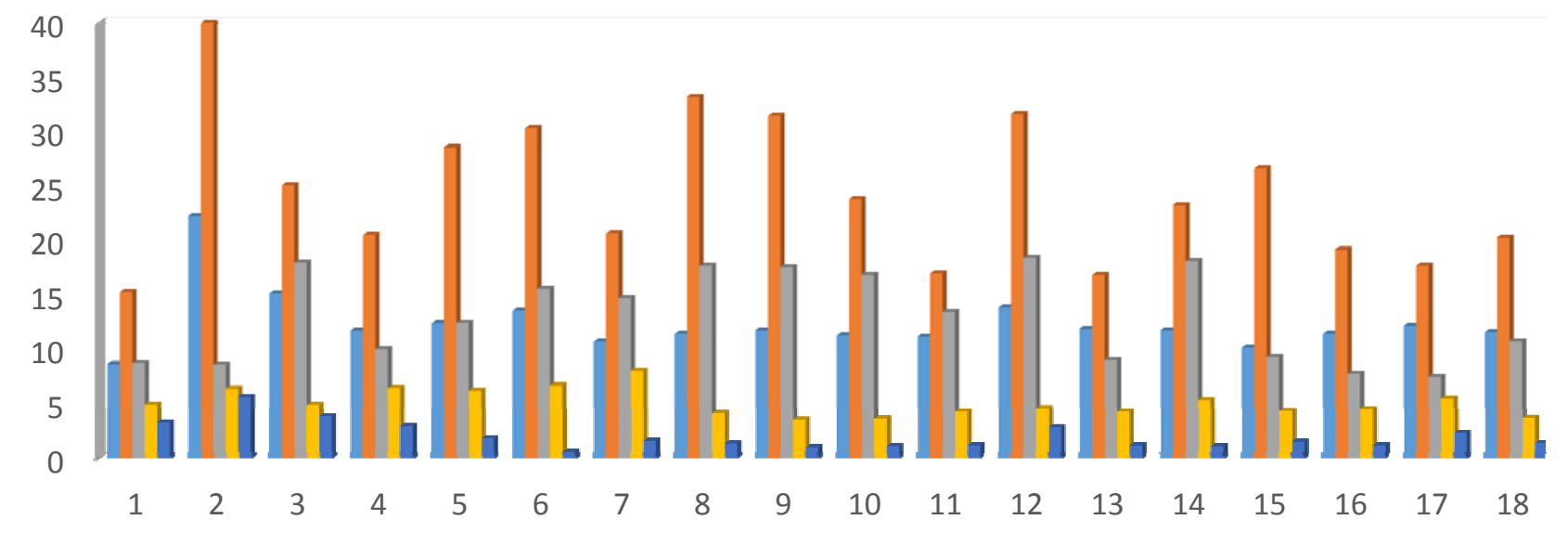

FIGURE II. LAB COLOR SPACE POLYNOMIAL LINEAR REGRESSION AFTER COLOR DIFFERENCE COMPARISON

\section{DATA ANALYSIS}

The results show that the polynomial linear regression method has a certain effect on the color reduction [6], and after the fourth order reduction, the chromatic aberration decreases close to zero, indicating that the chromatic aberration is close to zero increased order, the better the reduction effect.

At the same time, also found that the first-order reduction effect even more than the digital camera directly to the measured value of the effect is worse, greater color. The analysis shows that the first order polynomial linear regression is only four kinds of basic data, the amount of data is too small and the error is large.

Therefore, the effect of the polynomial linear regression reduction method is related to the reduction order, which improves the reduction order and increases the amount of the color sample data, which can effectively improve the reduction effect and achieve the purpose of obtaining the standard value of the camouflage color by using the digital camera to obtain the target and background The color of the field to improve the efficiency of color collection.

\section{CONCLUSION}

Based on the shortcomings and difficulties of color standard value acquisition under the existing field conditions, this paper puts forward the method of collecting color standard value by digital camera, and according to the color image space of digital camera itself is not the standard color space, the polynomial linear regression method is used to color The results show that the polynomial linear regression method is effective and the reduction effect is improved with the increase of the reduction order. The results show that the linear regression method is effective good, fourth order when the two color is almost zero conclusion. In the digital camera color reproduction effect has made some research results, but in the color of the production is more rough, there may be uneven color distribution, and the number of color samples less than the color difference between the color, but also on the experimental impact, but also the need to establish a data-rich military standard color-like database, which is the future to study the direction.

\section{REFERENCES}

[1] V. Chikane, and S. F. Chiou, "Automatic while balance for digital still camera,” Journal of Information Science and Endineering, vol. 22, pp. 497-509, 2006.

[2] S. Okada, Y. Matsuda, T. Yamada and A. KobYshi, "System on a chip for digitial still camera," IEEE Transactions on Consumer Electronics, vol. 45, pp. 584-590, 1999.

[3] H. L. Kwai, and C. Oscar, "Automatic White Balancing Using Luminance Component and Standard Deviation of RGB Componts,” ICASSP, 2004, pp. 493-496.

[4] M. S. Yao, Hiher algebra, Shanghai: Fudan University Press, 2005, pp. 198-199.

[5] ISO 17321: Colour Characterization of DSCs Using Colour Targets and Spectral Mumination.

[6] F. J. J. Clarke, R. McDonald and B. Rigg, "Modification to the JPC79 colourdifference formula,”J Soc Dyers Colour, vol. 100, pp. 128-132 and 281-282, 1984. 